\title{
Fault diagnosis for tilting-pad journal bearing based on SVD and LMD
}

\author{
Zhang Xiaotao and Li Weiguang \\ School of Mechanical and Automotive Engineering, South China University of Technology, Guangzhou, 510640, China
}

\begin{abstract}
Aiming at fault diagnosis for tilting-pad journal bearing with fluid support developed recently, a new method based on singular value decomposition (SVD) and local mean decomposition (LMD) is proposed. First, the phase space reconstruction of Hankel matrix and SVD method are used as pre-filter process unit to reduce the random noises in the original signal. Then the purified signal is decomposed by LMD into a series of production functions (PFs). Based on PFs, time frequency map and marginal spectrum can be obtained for fault diagnosis. Finally, this method is applied to numerical simulation and practical experiment data. The results show that the proposed method can effectively detect fault features of tilting-pad journal bearing.
\end{abstract}

\section{Introduction}

Tilting-pad journal bearing applied widely in high-speed rotary machinery has such advantages as strong carrying capacity, long operational life span and small friction factor, swing effect of tilting-pad further improving the stability of vibration [1]. The mechanical fulcrum that makes the stability of the operation system decline is one of the deficiencies of traditional tilting-pad journal bearing. Therefore, it is necessary to develop new type of tilting-pad journal bearing [2].

This paper developed one kind of three-pad tiltingpad journal bearing with fluid support. A large-scale test bench is established to evaluate its performance. Acceleration sensors are put near the screw bolt of footing on the test bench to acquire the vibration signal

The vibration signal from tilting-pad journal bearing contains large amount of information about the operation state, represented as non-stability and multi-component modulation signal. Moreover, this vibration signal contains large mutation and short-term shock component, which makes it hard to discriminate, particularly for the weak fault signal interfered by the surrounding equipment in the field. How to extract the fault feature from the interfered vibration signal is the key for the development of tilting-pad journal bearing.

There are many signal processing techniques that can extract the fault information from the vibration signal, windowed Fourier transform (WFT), wavelet transform and et al. Each of them has their own limitations. For WFT, once the window function is fixed, the size of the time-frequency window is unalterable, the time and frequency are the same for all the components that include different time scales [3]. Wavelet analysis could provide local features in both time and frequency domains and has the feature of multi-scale, which enables wavelet analysis to distinguish the abrupt components of the vibration signal [4]. Thus, wavelet analysis has been widely applied for fault diagnosis of rotary machinery [5]. However, wavelet analysis is essentially an adjustable window Fourier transformation. When a signal is decomposed by wavelet, only the rectangular timefrequency partition of time-frequency plane can be obtained and such tile partition would fail to guarantee instantaneous frequencies of each resulting component to have physical significance. Wavelet analysis has no selfadaptive feature in nature [6].

Empirical Mode Decomposition (EMD) is a selfadaptive signal processing method, which has been widely used for mechanical fault diagnosis and other fields [7]. However, there still exist many drawbacks in EMD such as the end effect, mode fixing, IMF stopping criterion, the problem of quick algorithm and envelope line, which are still underway.

The local mean decomposition (LMD) was proposed by Jonathan S. Smith [8] to decompose an amplitude and frequency modulated signal into a small set of product functions (PFs). The LMD method can be applied to analyse on wide variety of natural signals such as electrocardiograms, functional magnetic resonance imaging data, and earthquake data [9]. Here, we introduce the LMD method into tilting-pad journal bearing fault diagnosis.

SVD method has been widely applied in many fields during recent years, such as adaptive filter [10], noise reduction [11], etc. Therefore, in this paper SVD is used for noise reduction for vibration signal to get relatively purified signal before LMD decomposition.

This paper is organized as follows. Section 2 describes the theory of tilting-pad journal bearing with fluid support and tilting-pad journal bearing test bench. In section 3, the theory of SVD and LMD are introduced. In 
section 4, a simulation signal is processed to evaluate the proposed method. In section 5, the real vibration signal from the tilting-pad journal bearing test bench is tested. Finally, the conclusion is drawn in section 6.

\section{Tilting-pad journal bearing}

\subsection{The principle}

The structure of tilting-pad journal bearing is shown in Fig. 1. The whole bearing includes several pads. Each pad can swig on its mechanical pivot to adjust its position freely along with the axis movement to form wedge, adapting to the change of velocity and bearing load. The resultant force of oil-film pressure in each pad passes through the centre of axle journal of the rotor, not producing the tangential force that can cause instability. Theoretically tilting-pad journal bearing is fully stable. Therefore, it has been widely used in large rotating machinery, such as steam electric generating set, wind turbine and etc. [12]. However, its deficiencies in pad vibration and pivot friction damping will reduce working life and increase system instability.

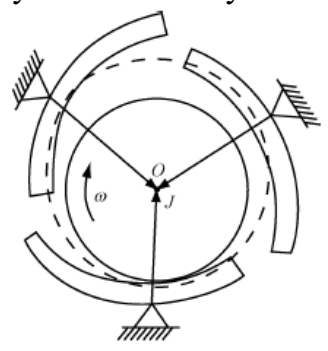

Figure 1. Schematic drawing of tilting-pad journal bearing with three mechanical pivots.

Three pads tilting-pad journal bearing with fluid support is developed in this paper, fluid support substituted for the mechanical pivot, being avoid of mechanical pivot friction and pad vibration. Tilting-pad journal bearing with fluid support has two layers of oil film, inner layer oil film and outer layer oil film. The inner oil film works as dynamic pressure oil film, the outer oil film works as static oil film or squeeze oil film.

\subsection{Test bench}

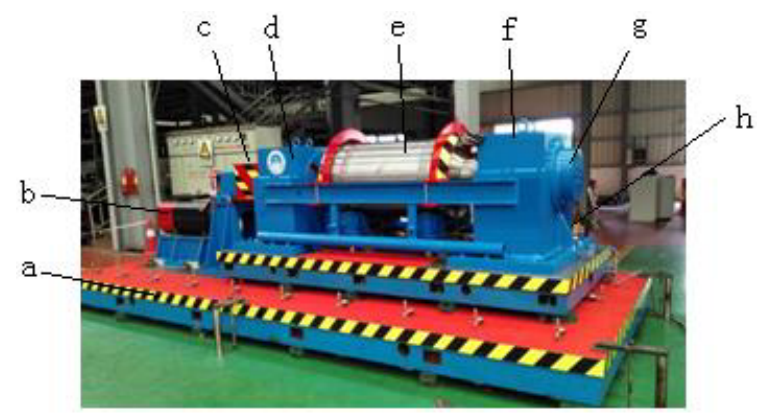

Figure 2. Tilting-pad journal bearing test bench. (a) Cast iron base. (b) Power servo motor. (c) Shaft coupling. (d) Front tilting-pad journal bearing. (e) Rotor. (f) Rear tilting-pad journal bearing. (g) Anti-thrust bearing. (h) Lubrication oil system.
A tilting-pad journal bearing test bench is built to evaluate the performance of the tilting-pad journal bearing as shown in Fig. 2.

The main parameters of the tilting-pad journal bearing test bench system are listed in table 1 .

Table 1. Main parameters of the test bench.

\begin{tabular}{l|l}
\hline Working bearing & $\begin{array}{l}\text { Tilting-pad journal bearing, } \\
\text { circular journal bearing }\end{array}$ \\
\hline Journal diameter of rotor & $\Phi 200 \mathrm{~h} 6(\mathrm{~mm})$ \\
\hline Working velocity range & $0 \sim 5000(\mathrm{RPM})$ \\
\hline Rotor quality & $4975 \mathrm{~kg}$ \\
\hline Input oil temperature & $30-45^{\circ} \mathrm{C}$ adjustable \\
\hline Oil feed pressure & $0 \sim 2 \mathrm{MPa}$ \\
\hline Lubrication oil & $68 \#$ turbine oil, $0.06052 \mathrm{~Pa} . \mathrm{s}$ \\
\hline Servo motor & 3-phase $\mathrm{AC}$ motor, $200 \mathrm{kw}$ \\
\hline
\end{tabular}

\section{Analysis methods}

\subsection{Principle of SVD}

Based on phase space reconstruction theory, the acquired signal expressed as $x=\left(x_{1}, x_{2}, \ldots, x_{N}\right)$ is constructed to be Hankel matrix $A$

$$
A=\left[\begin{array}{cccc}
x_{1} & x_{2} & \ldots & x_{q} \\
x_{2} & x_{3} & \ldots & x_{q+1} \\
\ldots & \ldots & \ldots & \ldots \\
x_{p} & x_{p+1} & \ldots & x_{N}
\end{array}\right]
$$

where $A$ is $p \times q$ order matrix, $N$ is the signal length, $N=p+q+1$.

Conduct SVD and we get

$$
A=U D V^{T}
$$

Where $U$ and $V^{T}$ are $p \times p$ and $q \times q$ orthogonal matrix respectively, $D$ is $p \times q$ diagonal matrix, $D=\operatorname{diag}\left(\lambda_{1}, \lambda_{2}, \ldots, \lambda_{k}, O\right), k=\min (p, q), O$ is the zero matrix, $\lambda_{i}(i=1,2, \ldots, k)$ are called the singular values of $A$. According to the theory of SVD and matrix optimal approximation under Frobenious normal, the previously bigger singular values reflect the useful signal, while the smaller singular values reflect the noise. If we set these smaller singular values to zero, the noise of acquired signal will be removed, then we reconstruct the signal with the rest singular values, we can get the denoised signal.

\subsection{Principle of LMD}

To extract fault information from a vibration signals, LMD decomposes the raw signal into a series of product functions (PFs). A PF, which is the product of an envelope signal and a frequency modulated signal, has actual physical significance. Based on the moving average method, LMD progressively smooth the signal. The details about LMD algorithm can be found in Refs. 
$[8,9]$. Based on the decomposition process, the original signal can be reconstructed by

$$
x(t)=\sum_{i=1}^{k} P F_{i}(t)+U_{k}(t)
$$

That is, the raw signal $x(t)$ can be reconstructed by all the PFs and a monotonic function $U_{k}(t)$. LMD method can guarantee the information integrity of the raw signal and effectively extract useful and comprehensive fault information.

\section{Simulation signal analysis}

A modulation signal $x(t)$ is shown in Fig. 3 to verify the proposed method, data length 1000, Hankel matrix dimension $501 \times 500$.

$$
x(t)=(1+1.5 t) \cos (30 \pi t)+2 \sin (70 \pi t)+\eta(t)
$$

where $\eta(\mathrm{t})$ reprents the additive Gaussian white noise,standard deviation 1.5.

Fig. 4 shows the singular values (SVs) distribution curve. According to singular values difference spectrum shown in Fig. 5, choose the second peak for noise reduction, the reconstructed signal is given in Fig. 6. The result of the reconstructed signal after LMD is shown in Fig. 7. From Fig. 7, it can be clearly seen that PF1 represents $35 \mathrm{~Hz}$ sinusoidal frequency and PF2 represents another modulation signal, which shows that SVD method can remove noise effectively and be helpful for LMD result explicable.

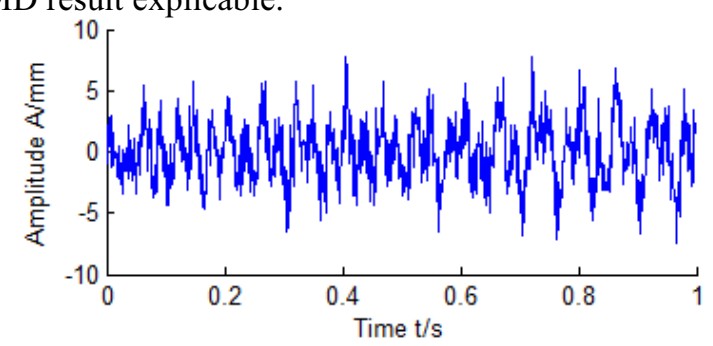

Figure 3. Simulation signal.

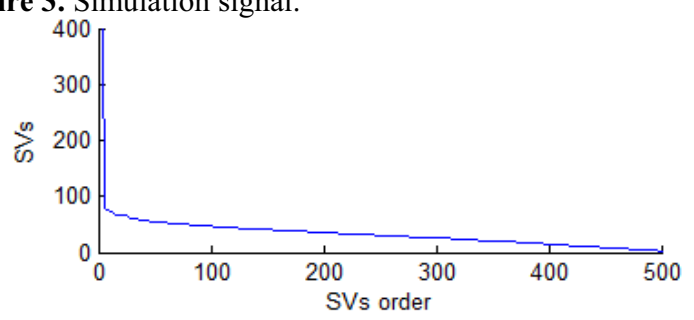

Figure 4. Singular values distribution curve of simulation signal.

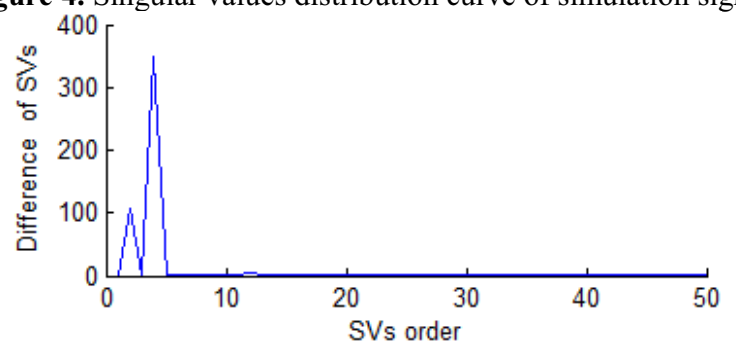

Figure 5. Difference spectrum of singular values.

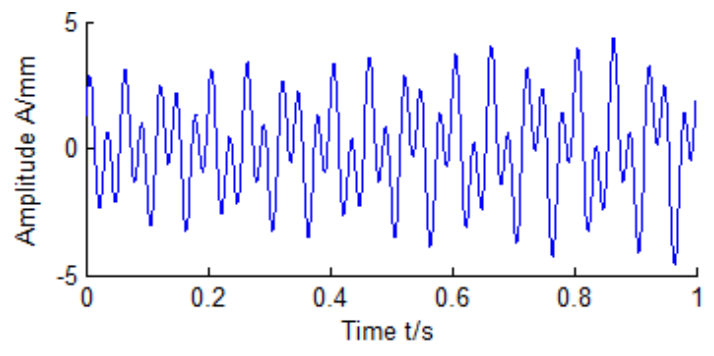

Figure 6. Reconstructed signal with the noise removed.
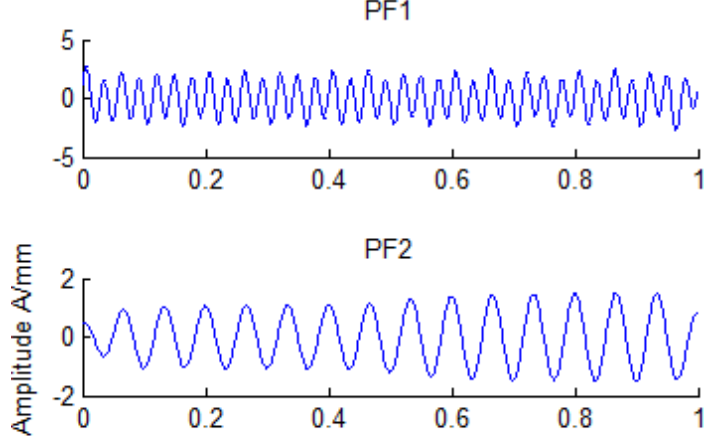

u2

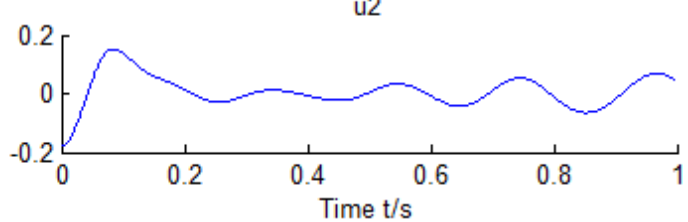

Figure 7. LMD result of the reconstructed signal.

\section{Experiment research}

\subsection{Test bench}

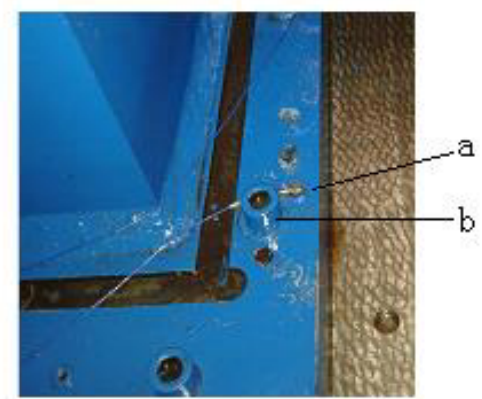

Figure 8. PCB accelerometers located along connecting bolts.(a) PCB accelerometers.(b) Connecting bolts.

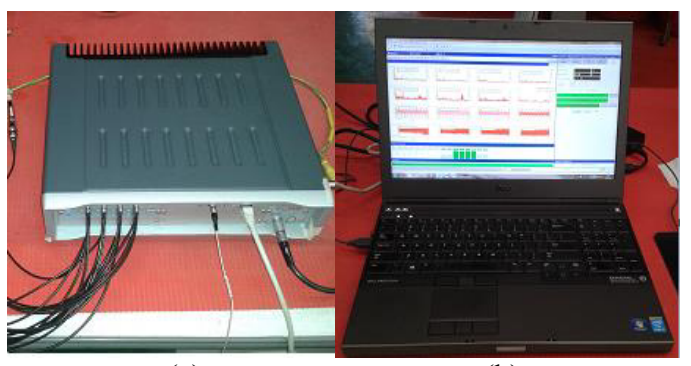

(a) (b)

Figure 9. LMS data acquisition system, (a) Hardware, SCADAS SCM202. (b) Software, Test.Lab 14A. 
Accelerometer is placed alongside connecting bolts as shown in Fig. 8. The model of accelerometer is PCB $333 \mathrm{~B} 32$ single axis, sensibility $100 \mathrm{mV} / \mathrm{g}$, measurement range $\pm 50 \mathrm{~g}$ p-p. Data acquisition system is shown in Fig. 9.

\subsection{Data analyses}

The tilting-pad journal bearing test bench works at $3900 \mathrm{r} / \mathrm{min}$, collect signal with the data length 1024 by $2048 \mathrm{~Hz}$, shown in Fig. 10.

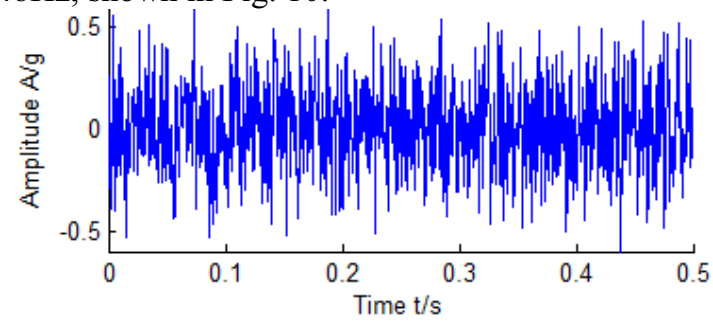

Figure 10. Collected signal from test bench.

As can be seen from Fig. 10, there is much noise which affects the following analysis. Thus, the method based on SVD is applied for noise reduction. Singular values distribution curve is shown in Fig. 11. According to the singular values difference spectrum shown in Fig. 12 , we choose 13 orders for noise deduction, and then the reconstructed signal is shown in Fig. 13.

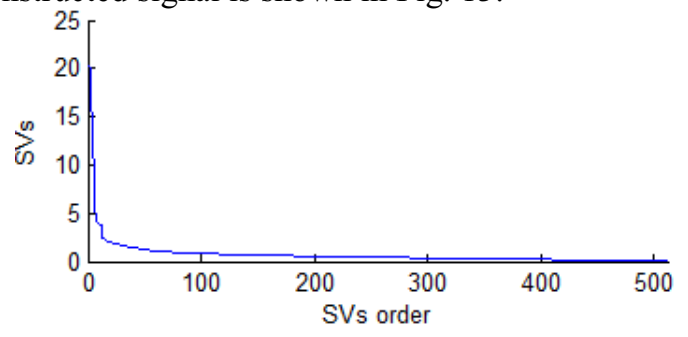

Figure 11. Distribution curve of singular values.

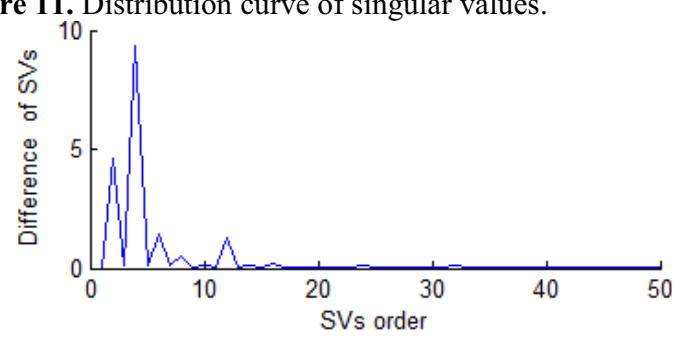

Figure 12. Difference spectrum of singular values.

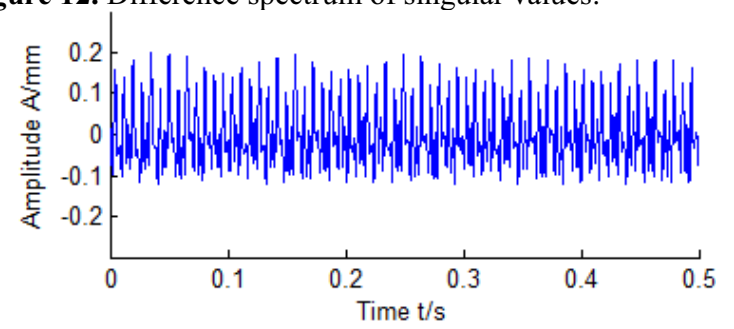

Figure 13. Restructured waveform.

From the comparison between Fig. 10 and Fig. 13, the noise is reduced effectively. Conduct LMD, the result is shown in Fig. 14.
PF1
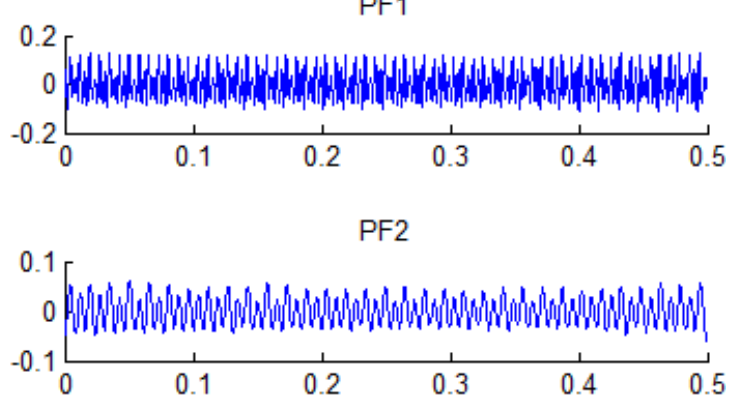

PF3

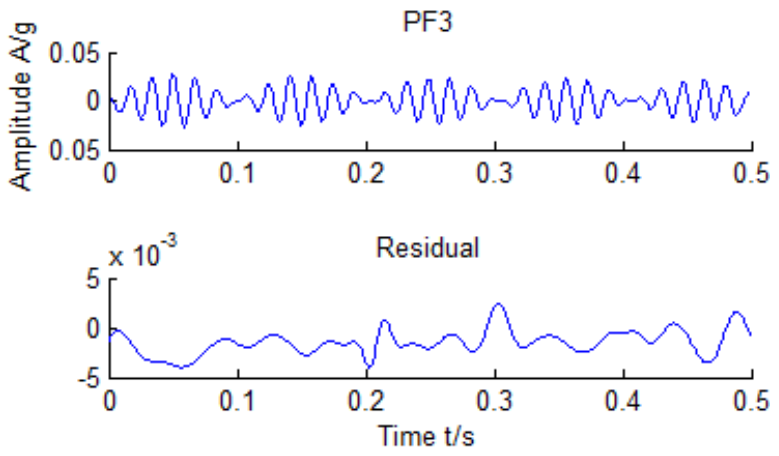

Figure 14. LMD Result of the signal after SVD denoising.

The useful PFs have strong correlation with the signal, while the useless PFs don't. Mutual information and correlation coefficient, which is used here, are common two methods to distinguish between these two kinds of PFs. The bigger the correlation between the PFs and original signal $x$ is, the bigger the correlation coefficient $\rho$ is. Correlation coefficient is defined as below:

$$
\rho_{x, P F_{i}}=\frac{\sum_{k=1}^{N} x(k) P F_{i}(k)}{\left[\sum_{k=1}^{N} x^{2}(k) \sum_{k=1}^{N} P F_{i}^{2}(k)\right]^{1 / 2}}
$$

The values of correlation coefficient between these PFs and signal before LMD are listed in Table 2, the value between the residual and the signal is also listed for comparison. From this table, we find the values of correlation coefficient between the PFs and the signal before LMD are far bigger than the value between the residual and the signal, which demonstrates that these PFs all are useful components.

Tab1e 2. Correlation coefficient values.

\begin{tabular}{lcccc}
\hline Component & $\mathrm{PF}_{1}$ & $\mathrm{PF}_{2}$ & $\mathrm{PF}_{3}$ & Residual \\
\hline $\begin{array}{l}\text { Correlation } \\
\text { coefficient }\end{array}$ & 0.845 & 0.591 & 0.236 & 0.015 \\
\hline
\end{tabular}

As can be seen from Fig. 14 the production functions (PFs) are non-stable, showing typical features of amplitude modulation and frequency modulation caused by the rubbing between rotor and stator [13].

Time-frequency map and marginal spectrum are shown in Fig. 15 and Fig. 16 respectively. As can be seen from Fig. 15, frequency varies dramatically with time. In Fig. 16, it is found there is wide range of spectrum around wording frequency $(65 \mathrm{~Hz})$ and its frequency 
multiplication. Large amount of experiments shows that vibration produced by friction from mechanical equipment will cause nearly continuous spectrum in some bands. The continuous frequency band is the characteristic of friction fault [14].

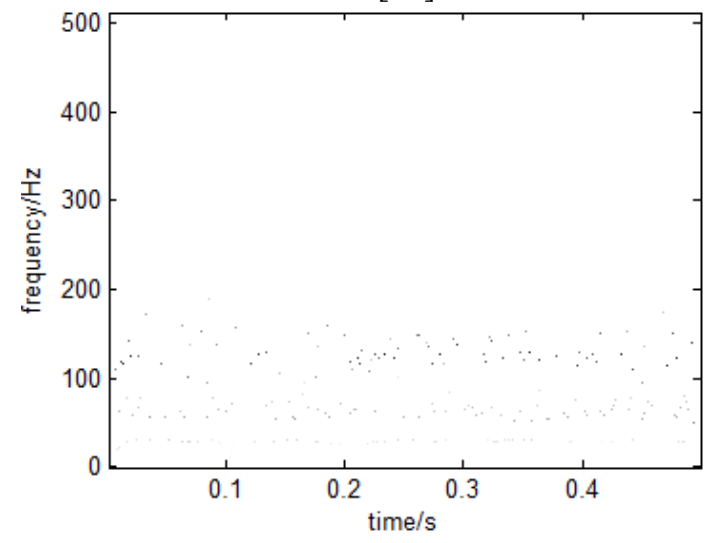

Figure 15. Time-frequency map.

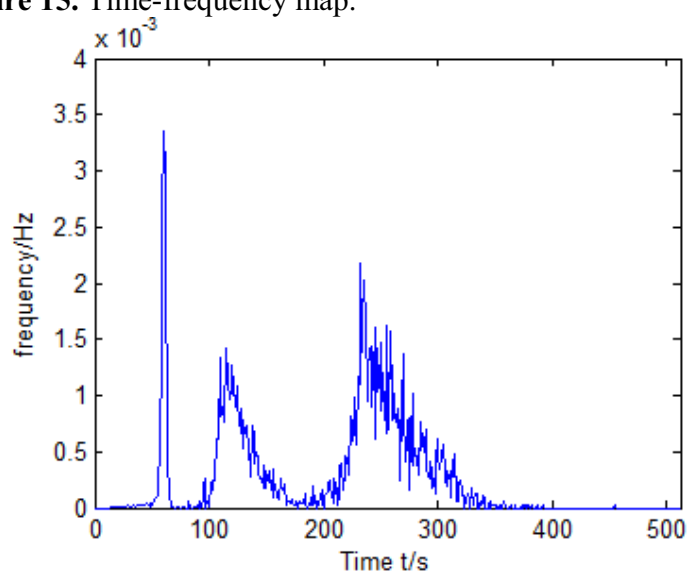

Figure 16. Marginal spectrum.

In order to verify this conclusion, stop the test bench and check the inner surface of the bottom pad of one tilting-pad journal bearing, find that there exists serious friction on it, as shown in Fig. 17.

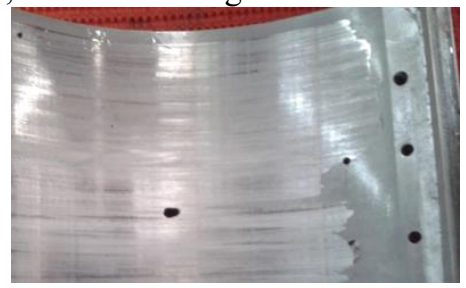

Figure 17. Inner surface of bottom pad of tilting-pad journal bearing.

\section{Conclusion}

Aiming at the fault diagnosis for the newly developed tilting-pad journal bearing with fluid support, this paper proposed the hybrid method based on SVD and LMD. Its efficiency has been evaluated on simulation analysis and experiment signal. The result demonstrates that the proposed method is feasible and effective for the fault diagnosis of the tilting-pad journal bearing with fluid support.
Tilting-pad journal bearing with fluid support is a new type of bearing, until now its theory foundation is still insufficient, which is an important project for future study. The reconstruction order of SVD has a great effect on the reconstructed signal and following signal analysis, so how to select effective reconstruction order may be another issue for investigation later.

\section{References}

1. L.Weiguang, Z. Xiaotao. Analysis on Static Performance of Tilting Pad Sliding Bearing. Lubrication Engineering 38(8), 6-12 (2014).

2. L. Suya. Bearing tester and testing technique. Bearing 8, 55-59 (2011)

3. Y.B. Fu, C.K. Chui, C.L. Teo. Accurate twodimensional cardiac strain calculation using adaptive windowed Fourier transform and Gabor wavelet transform. Int. J. Comput. Assisted Radiol. Surg. 8(1), 135-144(2013).

4. S. Mallat. A theory for multi-resolution decomposition, the wavelet representation. IEEE Trans. Pattern Anal. Mach. Intell. 11(7), 674 689(1989)

5. Z.K. Peng, F.L. Chu. Application of the wavelet transforms in machine condition monitoring and fault diagnostics: a review with bibliography. Mech. Syst. Signal Process. 18 (2), 199-221(1989).

6. S. Olhede, A.T. Walden. The Hilbert spectrum via wavelet projections. Proc. R. Soc. Lond 460, 955975(2004).

7. J.S. Cheng, D.J. Yu, Y. Yang. Energy operator demodulating approach based on EMD and its application in mechanical fault diagnosis. Chinese $\mathrm{J}$. Mech. Engrg. 40(8), 115-118(2004).

8. Smith Jonathan S. The local mean decomposition and its application to EEG perception data. J. Roy. Soc. Int. 2, 443-454(2005).

9. P. Cheolsoo, L. David, et al. The complex local mean decomposition,Neuro-computing. Neuro-computing, 74(6) ,867-875(2011).

10. M. R. Bai, S.J. Elliott. Preconditioning multichannel adaptive filtering algorithms using EVD- and SVDbased signal prewhitening and system decoupling.J. of Sound and Vibration, 270(4), 639-655(2004).

11. L. Lehtola, M. Karsikas, M. Koskinen, et al. Effects of noise and filtering on SVD-based morphological parameters of the T wave in the ECG. J. of Med. Eng. and Tech., 32(5), 400-407(2008).

12. Y. Lihua, Q. Shemiao, G. Haipeng, et al. The numerical analysis on the load capacity of aerodynamic tilting-pad journal bearings.Lubrication Engineering 32(4), 14-16(2007).

13. L. Luping, Z. Xinyuan, C. Jian. Typical features study on rubbing fault of steam turbine unit. J. of Vib. Mea. \& Diag. 12(4), 281-285(2001).

14. H. Zhengjia, Z. Yanyang, M. Qingfeng, et al. Fault diagnosis theory and application of non-stationary signal of mechanical equipment. (Beijing:Higher Education Press, 2001). 